Pramāna, Vol. 16, No. 2, February 1981, pp. 151-154. (c) Printed in India.

\title{
Electrical resistivity studies of benzidine-TCNQ and its inclusion compound under high pressure
}

\author{
A K BANDYOPADHYAY and S V SUBRAMANYAM \\ Department of Physics, Indian Institute of Science, Bangalore 560 012, India \\ MS received 24 November 1980; revised 13 January 1981 \\ Abstract. Electrical resistivity studies of the charge transfer complex benzidine- \\ TCNQ and its inclusion compound, have been carried out up to pressures $8 \mathrm{GPa}$. \\ Two types of behaviour were observed in these complexes under high pressure and \\ this difference is interpreted and discussed.
}

Keywords. Electrical resistivity; organic compounds; high pressure; benzidineTCNQ.

\section{Introduction}

It has been reported by Ohmasa et al (1971) that the acceptor tetracyanoquinodimethane (TCNQ) forms two types of compounds with aromatic donor benzidine (Bine), one containing the solvent molecule along with the complex and the other is solvent-free complex. The x-ray diffraction studies of the single crystals of these complexes clearly indicate (Ike-moto et al 1972, Yakushi et al 1974) that although both these compounds crystallize in monoclinic structure, in the case of solvent containing compound additional solvent molecules are found in the lattice. The relative orientation of the donor and the acceptor molecules is the same in both the crystals. However in the solvent-free molecule, the molecular columns are closely packed without giving any space for the solvent molecule. Thus in this molecule this charge transfer interaction is confined between the donor (Bine) and the acceptor (TCNQ). On the other hand in the solvent containing molecule, there exist two kinds of interactions-one is the usual charge transfer interaction and the other is the interaction due to the presence of the solvent molecule. Because of this difference in their nature, the electrical, magnetic and optical properties of these two complexes are quite different.

The present work has been undertaken to investigate the effect of high pressure on the electrical resistivity of these two complexes.

\section{Experimental}

It has been mentioned by Ohmasa et al (1971) that when the crystals are grown in a solvent. like dichloromethane, the complex contains the solvent molecule (BineTCNQ(S)) while the chloroform medium usually gives solvent-free crystals (Bine- 
TCNQ(N)). Thus dichloromethane and chloroform were used as the solvents for growing these crystals. The room temperature resistivity was found to be $\sim 10^{3}$ $\mathrm{ohm} \mathrm{cm}$ for Bine-TCNQ(S) and $\sim 10^{8} \mathrm{ohm} \mathrm{cm}$ for Bine-TCNQ(N). The crystals were characterized by first examining the composition through elemental analyses and then the optical absorption spectra. The characteristic first and second charge transfer bands are in good agreement with the earlier reported value (Amano et al 1969). The x-ray diffraction pattern for powder samples were taken to verify the crystal structure of these two crystals. The powder pattern agrees quite well with the reported pattern by Ohmasa et al (1971).

The high pressure experiments were carried out in a clamp-type high pressure cell. The cell consists of a pair of Bridgman anvils made of tungsten carbide with 3\% cobalt binder and has a tip diameter $4 \mathrm{~mm}$. Steatite is used as the pressure transmitting medium and heat-treated pyrophyllite as the gasket material. The details of high pressure arrangement and calibration values are published by Bandyopadhyay et al (1980a).

Experiments were carried out on both the single crystals and the compacted pellets. The pellets were made by powdering and pressing them in a die. The conventional four-probe and two-probe methods have been used for these measurements with a Keithley constant current source (model no. 225) and a digital electrometer (model no. 616) as the measuring instruments.

\section{Results and discussion}

Figures 1 and 2 show the resistivity versus pressure curves for Bine-TCNQ(S) and

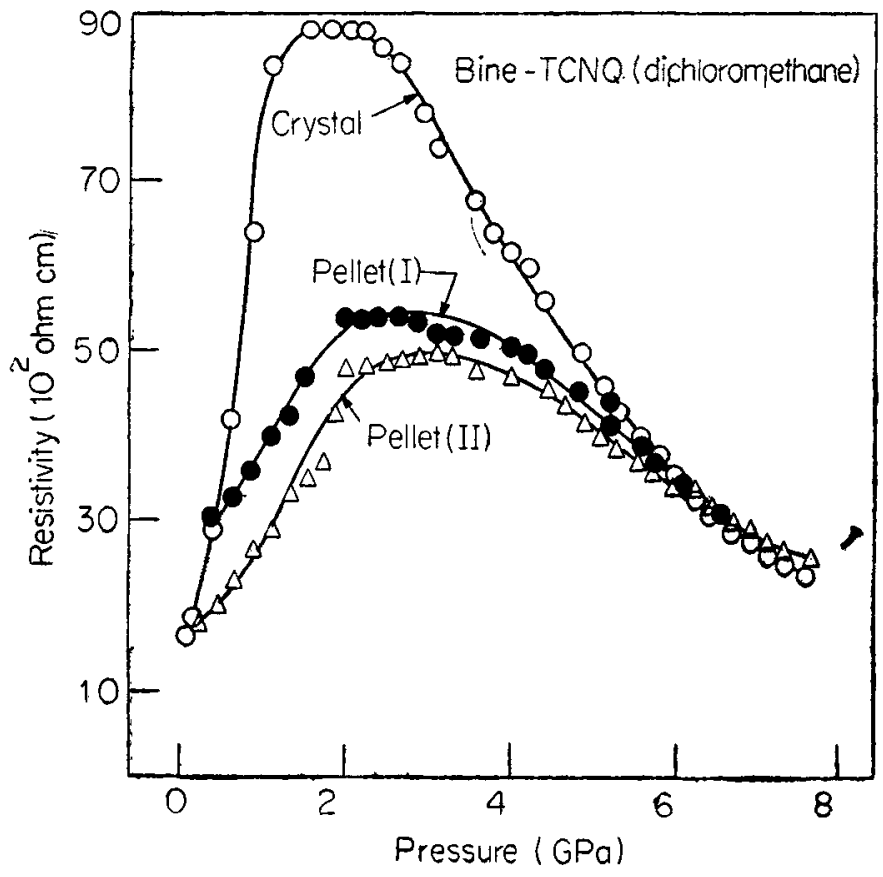

Figure 1. Resistivity versus pressure curve for Bine-TCNQ(S). 


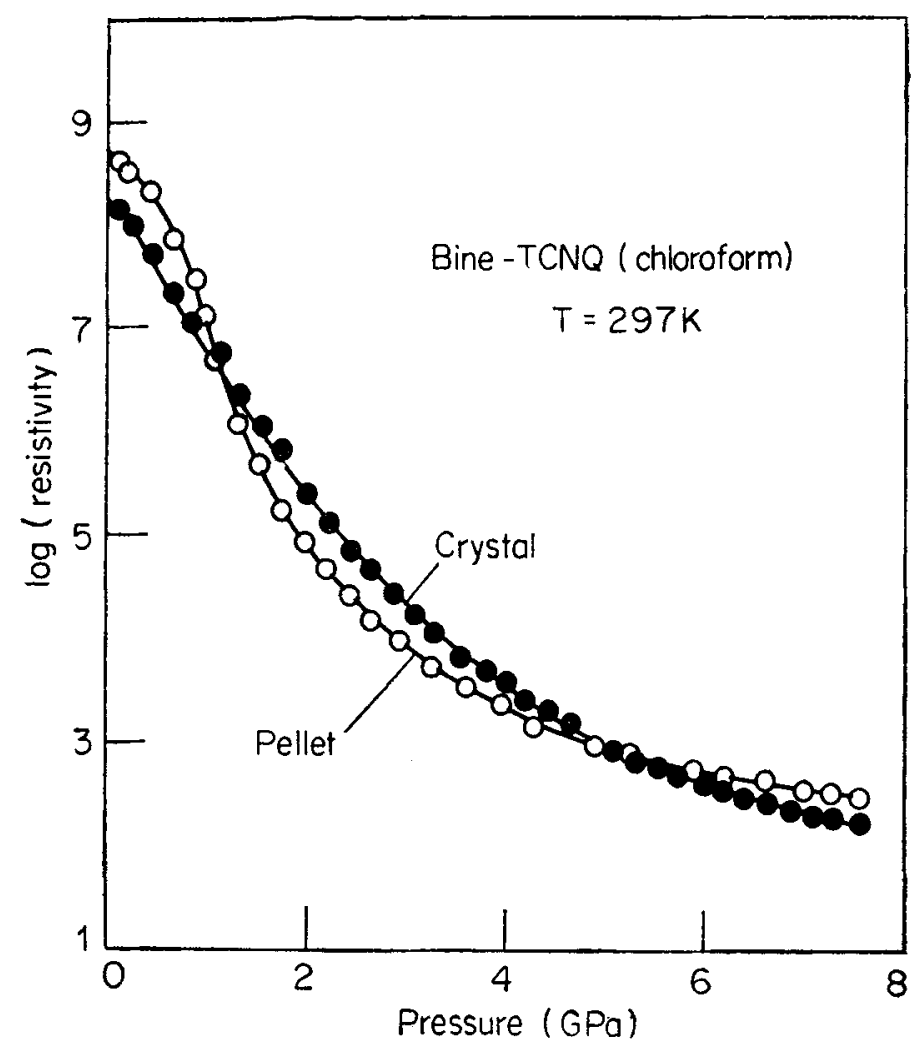

Figure 2. Resistivity versus pressure curve for Bine-TCNQ(N).

Bine-TCNQ(N) on both the single crystals and pellets. In the case of Bine-TCNQ(S), the resistivity increases with the increasing pressure and reaches a maximum around $2.0 \mathrm{GPa}$ and then starts decreasing. While in Bine-TCNQ(N), there is strong negative pressure derivative of resistivity in the initial pressure range but at higher pressures, there is a marked tendency towards saturation.

This entirely different behaviour of these two systems under high pressure is not very clear. In Bine-TCNQ(N) the observed behaviour can be interpreted in the framework of our earlier proposed model (Bandyopadhyay et al 1980b), where it was assumed that the enhancement of pressure causes the interatomic distance to decrease and this results in the increase of molecular overlap. This overlap increases linearly with pressure in the low pressure region but as the pressure increases, the steric hindrance between the two molecules dominates preventing further decrease of the distance. Thus as the pressure increases slow stiffening effect predominates. We have calculated the variation of resistivity with pressure in the tight binding approximation. It is shown there that the resistivity can be expressed as,

$$
\rho / \rho_{0}=\exp \left(A P+B P^{2}+C P^{3}\right)
$$

where $A, B$ and $C$ are constants related to the elastic constants of the system. An attempt has been made to fit our data with equation (1). It turns out that the fitting 
is quite satisfactory. The values of the constants are $A=-1.81226(\mathrm{GPa})^{-1}$, $B=1.67271 \times 10^{-1}(\mathrm{GPa})^{-2}$ and $C=-4.63383 \times 10^{-3}(\mathrm{GPa})^{-3}$.

However, in the case of solvent containing compound Bine-TCNQ(S), the increase in resistivity is rather unusual. It has been mentioned that this solvent molecule goes inside the lattice and forms an inclusion compound. Thus the low resistivity of this compound at normal atmospheric pressure is probably due to the presence of this solvent molecule. But as the pressure increases, the solvent molecules are likely to move out from the conducting strand. This will give rise to the interruption of the conducting strand and results in the increase in resistivity. However, this process will not continue for a long time because above a certain pressure, the solvent molecule comes out totally from the stack leaving the donor and acceptor alone. Above that pressure, the resistivity will decrease with increase of pressure as has been observed in the solvent-free complex. Thus the resistivity peak which has been observed in the present case can be attributed to the manifestation of this type of effect. For further verification, we have carried out low temperature studies at different clamped pressures on both the samples (not shown here). We observed, in Bine-TCNQ(N), the activation energy decreases with pressure while in Bine$\mathrm{TCNQ}(\mathrm{S})$, the activation energy increases with pressure and reaches a maximum at around $2.0 \mathrm{GPa}$ and then decreases accordingly. So this once again gives further support to the present model. In our earlier electrical resistivity studies under high pressure on the inclusion compound of poly-iodine chain, like a-cylodextrin-KI- $\mathrm{I}_{2}$, etc. (Oza et al 1978), we observed the same sort of behaviour as has been observed in Bine-TCNQ(S). It was found that the increase in resistivity with pressure is not due to ionic origin but due to some mechanism of electronic origin. So it is tempting to say that the same sort of mechanism is also responsible for these complexes under high pressure.

\section{Acknowledgement}

Part of the work was carried out during the authors' stay at the International Centre for Theoretical Physics, Trieste (Italy). They would like to acknowledge the help of Prof. M Tosi. Financial assistance from the Department of Science and Technology, New Delhi is gratefully acknowledged.

\section{References}

Amano T, Kuroda H and Akamatu H 1969 Bull. Chem. Soc. Jpn. 42671

Bandyopadhyay A K, Nalini A V, Gopal E S R and Subramanyam S V 1980a Rev. Sci. Instrum. 51136

Bandyopadhyay A K, Chatterjee S, Subramanyam S V and Bulka B R 1980b J. Phys. C13 L803

Ikemoto I, Chaikaishi K, Yakushi K and Kuroda H 1972 Acta Crystallogr. B28 3502

Ohmasa M, Kinoshita M and Akamatu H 1971 Bull. Chem. Soc. Jpn. 44391 and references therein.

Oza A K T, Bandyopadhyay A K and Subramanyam S V, 1978 Proc. Nucl. Phys. Solid State Phys. Bhabha Atomic Research Centre, C21 168

Yakushi K, Ikemoto I and Kuroda H, 1974 Acta. Crystallogr. B30 835 